Revue bibliographique pour le domaine irano-aryen

\title{
Diana Edelman, Anne Fitzpatrick-Mckinley, Philippe Guillaume (eds.). Religion in the Achaemenid Persian Empire
}

\section{Astrid Nunn}

\author{
(2) OpenEdition \\ Journals \\ Édition électronique \\ URL : http://journals.openedition.org/abstractairanica/44001 \\ DOI : 10.4000/abstractairanica.44001 \\ ISBN : 1961-960X \\ ISSN : 1961-960X \\ Éditeur : \\ CNRS (UMR 7528 Mondes iraniens et indiens), Éditions de l'IFRI
}

\section{Référence électronique}

Astrid Nunn, « Diana Edelman, Anne Fitzpatrick-Mckinley, Philippe Guillaume (eds.). Religion in the Achaemenid Persian Empire », Abstracta Iranica [En ligne], Volume 37-38-39 | 2018, document 8, mis en ligne le 30 décembre 2018, consulté le 29 septembre 2020. URL : http://journals.openedition.org/ abstractairanica/44001; DOI : https://doi.org/10.4000/abstractairanica.44001

Ce document a été généré automatiquement le 29 septembre 2020.

Tous droits réservés 


\title{
Diana Edelman, Anne Fitzpatrick- Mckinley, Philippe Guillaume (eds.). Religion in the Achaemenid Persian Empire
}

\author{
Astrid Nunn
}

\section{RÉFÉRENCE}

Diana Edelman, Anne Fitzpatrick-Mckinley, Philippe Guillaume (eds.). Religion in the Achaemenid Persian Empire. Tübingen: Mohr Siebeck, 2016, 390 p. (Orientalische Religionen in der Antike 17), ISBN 978-3-16-153960-2

1 Ce volume est le résultat du workshop "Religion in the Persian Period: Emerging Judaisms and Other Trends" qui a eu lieu à l'université de Sheffield en juillet 2011. Quelques contributions y ont été ajoutées. Il complète A. Fitzpatrick-McKinley (éd.), Assessing Biblical and Classical Sources for the Reconstruction of Persian Influence, History and Culture. Harrassowitz, 2015.

2 Le but de ce workshop: gagner une vue plus large sur tous les courants religieux au sein de l'Empire achéménide et - en relation - les échanges culturels et la "tolérance perse" qui était en fait une stratégie politique.

3 La monographie comporte deux parties. La première intègre les articles traitant de "Trends in Emerging Judaism" (p. 9-133) et la seconde "Other Religious Trends in the Persian Empire" (p. 137-367).

4 Dans la première partie les contributions de James Anderson, Philip Davies, Russel Hobson, Philippe Guillaume et Lowell K. Handy sont axées sur la théologie. C'est la raison pour laquelle nous n'avons sélectionné que les quelques articles suivants. 
Christian Frevel et Katharina Psychny, "A Religious Revolution Devours Its Children: The Iconography of the Persian-Period Cuboid Incense Burners" reprennent le thème présenté à Bochum et publié en 2014. Leur présentation, cette fois-ci en anglais, joue sur les mêmes registres variés et possède la même qualité qu'en 2014.

La seconde partie débute avec la contribution de Anne Fitzpatrick-McKinley, "Continuity between Assyrian and Persian Policies toward the Cults of Their Subjects". Elle soutient que les Perses ne toléraient les diverses religions de leurs sujets que pour des raisons politiques. Sur ce point, ils ne se différenciaient pas des Assyriens qui comme les Achéménides n'étaient "ni entièrement agressifs, ni entièrement tolérants" (p. 164). L'attitude des deux "régimes" était beaucoup plus comparable contrairement à ce que la tradition des "Assyriens cruels et des Perses tolérants" a développé. Cette attitude de "tolérance" est en réalité le propre de rois autoritaires.

7 Damien Agiut-Labordere arrive au même résultat en décrivant les changements dans le culte des temples égyptiens qui suivirent l'arrivée de Cambyse. Outre certains changements fiscaux, le contrôle royal augmenta fortement tout en cherchant la coopération avec l'élite de Memphis. Jared Krebsbach pense également que la "tolérance" perse a permis au Grand Roi de prendre en Egypte le rôle du pharaon, défenseur de l'ordre mondial.

8 Jason Silverman argumente sur les frontières entre mythologie, religion et politique impériale dans la "theology of kingship" achéménide. Yannick Muller considère dans quelle mesure la pratique des mutilations dans l'Empire achéménide était liée à la religion.

Diana Edelman, "Iconography on Double-Shekel Sidonian Coinage in the Persian Period: Is it a God or a King in the Chariot?" reprend la question "classique" de savoir qui sont les deux personnages dans le char. Pour cela, elle analyse l'iconographie des monnaies sidoniennes en les divisant en quatre groupes chronologiques. Elle débat ensuite de l'influence perse et grecque sur ces motifs et des différents types de chars. En insistant sur le fait qu'aucun motif n'est sans contexte, que le motif du char a sans aucun doute été emprunté à l'Orient et qu'il y est aussi une "iconic royal Persian image", elle arrive à la conclusion que le second personnage dans le char ne peut être que le Grand Roi.

10 Mark Christian essaie de mesurer l'influence de la religion phénicienne sur la flotte perse par l'intermédiaire des marins phéniciens.

11 Enfin Deniz Kaptan examine les traditions religieuses en Anatolie achéménide à travers le matériel iconographique des bulles à empreintes sigillaires et des stèles en provenance de Daskyleion. Comme ailleurs, certains changements peuvent être observés sur un fond qui reste celui de la tradition et surtout celui de la diversité caractéristique de l'Anatolie. 


\section{AUTEURS}

\section{ASTRID NUNN}

Université de Munich 